\title{
CARACTERÍSTICAS PALINOLÓGICAS DE LAS ARALIÁCEAS DE MÉXICO*
}

\author{
VICTORIA SOSA**
}

\section{RESUMEN}

En este trabajo se analizan desde el punto de vista palinológico 17 especies de Araliaceae de México de los géneros Aralia, Dendropanax, Didymopanax, Hedera, Oreopanax y Sciadodendron. Para lograrlo se utilizó tanto microscopio electrónico de barrido (MEB) como microscopio de luz.

Las descripcione palinológicas se presentan a nivel genérico. Se incluye una clave de identificación de granos de polen para los diferentes géneros. Los resultados muestran que los granos de los distintos géneros son muy similares, aunque sí se encontraron diferencias especialmente en características de la exina, ya que Hedera y Didymopanax tienen granos semi-tectados, mientras que el resto los tienen tectado-perforados. Se compararon estas características palinológicas con otras morfológicas, encontrándose que muchos caracteres palinológicos considerados como primitivos coinciden con caracteres morfológicos de grupos de araliáceas primitivas.

\section{ABSTRACT}

This work studies 17 species of mexican Araliaceae from the palynological point of view belonging to the genera Aralia, Dendropanax, Didymopanax, Hedera, Oreopanax y Sciadodendron. All the pollen descriptions are presented at the generic level. The grains were studied based in scanning electron microscope and light microscope. It includes a dichotomous key for the genera grains. The results showed few differences between the pollen grains of the genera although some exine characters are important. Hedera and Didymopanax presented an exine semi-tectate and the rest of the genera had tectate perforate grains. This paper compared also some pollen characters with morphological

* Recibido para publicación en marzo de 1983.

**Instituto Nacional de Investigaciones sobre Recursos Bióticọs. Ap. Postal 281. 97000 Mérida, Yucatán.

Sosa V. 1983. Características palinológicas de las araliáceas de México. Boletín de la Sociedad Botánica de México 45: 117-132. 
ones, finding pollen characters considered primitive correlated with characters of primitive Araliaceae groups.

\section{INTRODUCCIÓN}

Los taxa de las araliáceas mexicanas se encuentran pobremente estudiados desde el punto de vista palinológico. En este trabajo se analiza la morfología de los granos de polen de este grupo de plantas ya que son importantes desde varios puntos de vista, como por ejemplo que aparezcan en los espectros polínicos fósiles de diversas regiones tanto tropicales como templadas. Graham \& Jarzen (1969) encuentran al género Dendropanax en el oligoceno de Puerto Rico; Gruas-Cavagnetto \& Bui (1976) encuentran Acanthopanax, Aralia, Brassaiopsis, Scheffleropsis y Panax en el paleoceno inglés y francés; Muller (1981) en su recopilacikón de polen fósil de angiospermas y en base a su recopilación bibliográfica afirma que el polen tipo Aralia se presenta desde el paleoceno en Europa y que el polen tipo Hedera se presenta desde el mioceno superior, en España y Alemania.

Además, algunas de las especies de araliáceas, como Dendropanax arboreus son melíferas (Cházaro, 1982).

Asimismo, en varios casos la morfología palinológica ha sido una herramienta valiosa en la determinación de las relaciones entre géneros de esta familia, posición sistemática de los géneros y también sus relaciones con otros taxa cercanos (Chao, 1954; Tseng, 1972).

Entre los estudios de morfología polínica que anteceden a este trabajo, podemos citar los de Straka \& Simon (1967) Erdtman (1972) y Radulescu (1974) que estudiaron la morfología del polen de algunas especies de araliáceas, básicamente europeas y asiáticas. Palacios (1968) describe la palinología de la especie Oreopanax peltatus para el estado de Morelos, México.

Desde el punto de vista palinológico, este artículo estudia 17 especies de los géneros silvestres Aralia, Dendropanax, Didymopanax, Oreopanax y Sciadodendron (que ha sido colectado recientemente en las costas del Pacífico) y el género Hedera ampliamente cultivado en México. Estudios florísticos previos (Smith, 1944 y Sosa, 1979) reportaron 16 especies silvestres de araliáceas para México.

Además de describir morfológicamente los granos de polen, este trabajo tiene como objetivos encontrar diferencias y similitudes entre los granos de los distintos géneros de araliáceas mexicanas; para ello trata de asociar estos caracteres con otros, como son las características morfológicas. Todas las descripciones palinológicas específicas se reunieron para presentarlas a nivel genérico.

\section{MATERIALES Y MÉTODO}

Las muestras de polen se tomaron de dos ejemplares para cada especie (excepto en los casos en los que sólo había un ejemplar disponible) de los herbarios del Instituto Nacional de Investigaciones sobre Recursos Bióticos (XAL), Escuela Nacional de Ciencias Biológicas del IPN (ENCB) y Herbario Nacional del Instituto de Biología de la UNAM (MEXU). En la tabla 1 se presenta la relación de especies con sus respectivos ejemplares. En este estudio quedaron excluidas tres especies de Oreopanax, O. langlassei Standley, O. sanderianus Hemsley y $O$. platyphyllus Marchal, que han sido reportadas 
TABLA 1. Especies de araliáceas estudiadas

\begin{tabular}{|c|c|c|}
\hline Especie & Ejemplares y Herbarios & Localidad \\
\hline Aralia humilis Cav. & $\begin{array}{l}\text { Webster y Kennedy } 17818 \text { (MEXU) } \\
\text { Rzedowski } 33954 \text { (ENCB) }\end{array}$ & $\begin{array}{l}\text { N de Tuxtla Gutiérrez, Chis. } \\
\text { Carretera Orizaba-Puebla }\end{array}$ \\
\hline A. racemosa $\mathrm{L}$. & Wendt y Adamcewitz 462 (ENCB) & Sierra Maderas del Carmen, Coah. \\
\hline A. regeliana Marchal & $\begin{array}{l}\text { McVaugh } 10348 \text { (MEXU) } \\
\text { Delgado } 329 \text { (MEXU) }\end{array}$ & $\begin{array}{l}\text { Pinal de Amoles, Qro. } \\
\text { Paso de León, Hgo. }\end{array}$ \\
\hline A. scopulorum Brand. & $\begin{array}{l}\text { Carter y Ferris } 3984 \text { (MEXU) } \\
\text { Carter y Sharsmith } 4165 \text { (MEXU) }\end{array}$ & $\begin{array}{l}\text { Sierra de la Giganta, BCS } \\
\text { Sierra de la Giganta, BCS }\end{array}$ \\
\hline D. gonatopodus (Donn. Smith) A.C. Smith & Miranda 7794 (MEXU) & Pueblo Nuevo, Chis. \\
\hline D. populifolius (Marchal) A.C. Smith & Miranda 1132 (MEXU) & Cuasimulco, Oax. \\
\hline Didymopanax morototoni (Aublet) Decne. \& Planchon & Vázquez et al. 1405 (XAL) & San Juan Guichicobi, Ver. \\
\hline O. echinops (Schlecht. \& Cham.) Decne. \& Planchon & Avendaño 571 (XAL) & Huatusco, Ver. \\
\hline O. flaccidus Marchal & Calzada 5479 (XAL) & Acajete, Ver. \\
\hline O. liebmannii Marchal & $\begin{array}{l}\text { Dorantes et al. } 1593 \text { (XAL) } \\
\text { Ventura } 9091 \text { (XAL) }\end{array}$ & $\begin{array}{l}\text { Acajete, Ver. } \\
\text { Acajete, Ver. }\end{array}$ \\
\hline O. obtusifolius L.O. Williams & $\begin{array}{l}\text { Vázquez et al. } 241 \text { (XAL) } \\
\text { Calzada y Cedillo } 68 \text { (XAL) }\end{array}$ & $\begin{array}{l}\text { Hidalgotitlán, Ver. } \\
\text { Los Tuxtlas, Ver. }\end{array}$ \\
\hline O. peltatus Linden & Dorantes 3539 (XAL) & Hidalgotitlán, Ver. \\
\hline O. xalapensis (H.B. \& K.) Decne. \& Planchon & $\begin{array}{l}\text { Beaman y Álvarez } 6453 \text { (XAL) } \\
\text { Avendaño } 69 \text { (XAL) }\end{array}$ & $\begin{array}{l}\text { San Martín Tuxtla, Ver. } \\
\text { Cerro Macuiltepetl, Ver. }\end{array}$ \\
\hline
\end{tabular}




\section{BOLETÍN DE LA SOCIEDAD BOTÁNICA DE MÉXIGO No. 45, 1983}

en la literatura pero sin materiales adecuados para preparar muestras palinológicas en los herbarios consultados.

Las muestras se dividieron en dos grupos. El primero no se trató químicamente y para observarlas en el microscopio electrónico de barrido, se sombrearon y cubrieron con Au para poder fotografiarlas.

El segundo grupo se trato químicamente, siguiendo la técnica de acetólisis de Erdtman (1943) haciendolo previamente con $\mathrm{KOH}$ al $10 \%$. Para poder estudiar estas muestras en microscopio electrónico de barrido, se pasaron por punto crítico y sombrearon con Au y observadas con un microscopio marca JEOL, Modelo JSMT-20. Se fotografiaron con placa Royal Pan, 400 ASA, imprimiéndose, por contacto, en papel Kodabromide F-4.

Los granos también se observaron al microscopio de luz y montaron en gelatina glicerinada. Para las descripciones palinológicas, se midieron y observaron al azar, 10 granos de cada ejemplar. En las fotografías de microscopio de luz, se usó un modelo Zeiss standard 7 con cámara adaptada y película Panatomic X; se imprimieron en papel Kodabromide F-4.

Las colecciones de laminillas y fotografías al MEB, material de referencia para este estudio, se encuentran depositadas en la Palinoteca de los herbarios XAL, ENCB y MEXU.

En las descripciones morfológico-palinológicas se utilizó la terminología basada en Erdtman (1952) y de la enciclopedia palinológica de Kremp (1965).

Los resultados se presentan como descripciones palinológicas a nivel de género, con los rangos de las principales características palinológicas. Tomando en cuenta que en la mayoría de los casos se midieron las muestras de dos ejemplares de herbario para cada especie. Se presenta también una clave de identificación a nivel palinológico.

\section{CLAVE DE IDENTIFICACIÓN DE LOS GRANOS DE POLEN DE LOS GÉNEROS DE ARALIÁCEAS MEXICANAS}

Granos con la exina semi-tectada

Granos no margo-colpados; muros simples; diámetro de las perforaciones sin formas regulares ........

Didymopanax (Fig. 3)

Granos margo-colpados; muros simples y duplibaculados; diámetro de las perforaciones con forma de polígonos irregulares

Hedera

(Fig. 2)

Granos con la exina tectado-perforada

Granos margo-colpados

Oreopanax

Aralia

(Fig. 5)

(Fig. 6)

Granos no margo-colpados

Granos por lo general subesferoidal a esferoidal

Sciadodendron

(Fig. 4)

Granos por lo general prolados

Dendropanax

(Fig. 1) 


\section{PALINOLOGÍA DE ARALIÁCEAS}

\section{DESCRIPCIONES PALINOLÓGICAS}

Aralia (Tournefort) L.

Especies estudiadas:

A. humilis Cav., (Fig. 6), A. racemosa L., A. regeliana Marchal (Figs. 1-5, 8), A. scopulorum Brand (Figs. 7, 9).

Granos eumónadas de simetría radial, isopolares.

Abertura tricolporada con un colpo transversal más o menos desarrollado. Exina tectadoperforada a microreticulada, margo-colpada, las lúminas de $2 \mu$ y los muros simples menores de $2 \mu$, su grosor de 4 a $8 \mu$. Perímetro en vista polar subangular.

Forma prolada subesferoidal a subprolada.
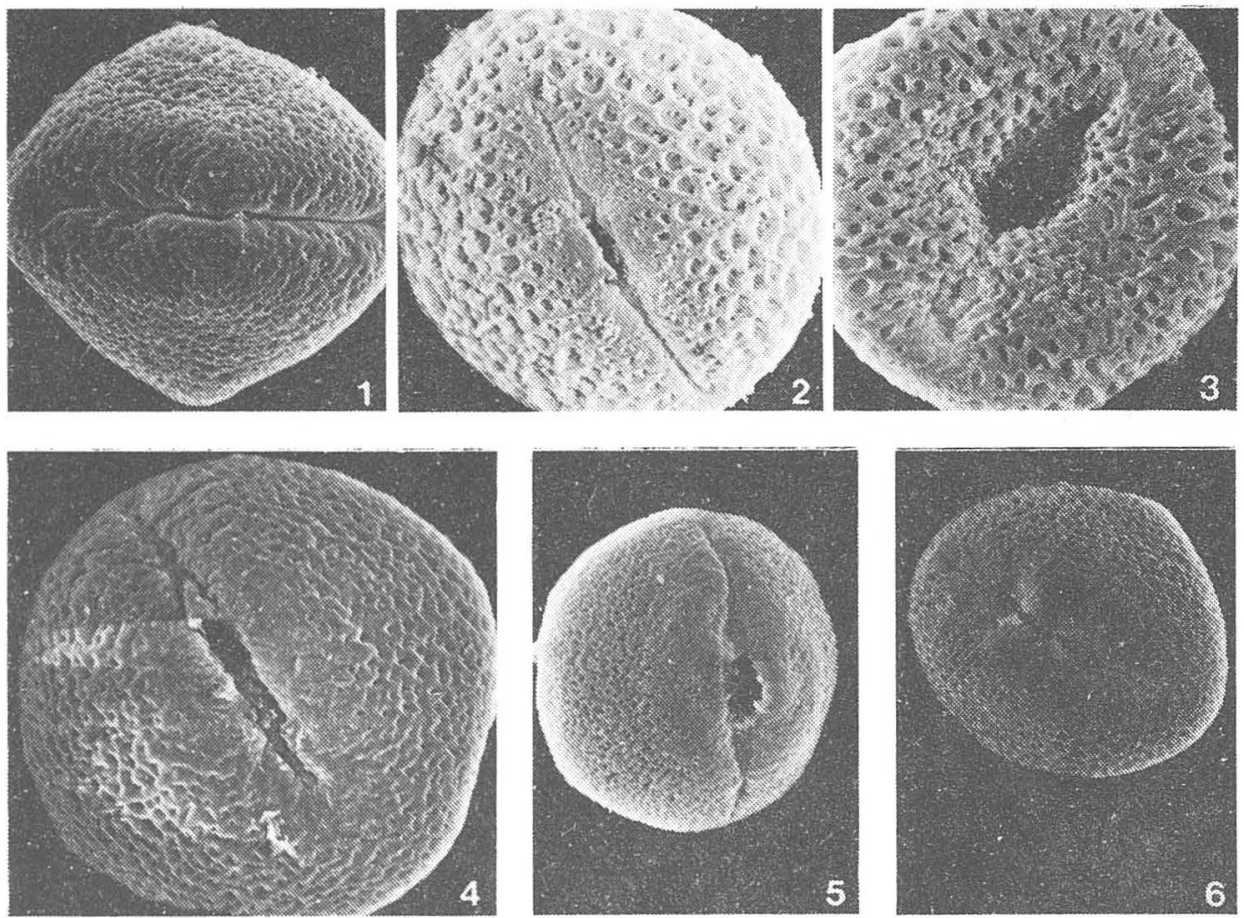

Lámina I. Figuras (Fotografías al MEB):

1. Dendropanax populifolius. Vista ecuatorial, grano acetolizado.

2. Hedera helix. Vista ecuatorial, grano acetolizado.

3. Didymopanax morototoni. Vista ecuatorial, grano acetolizado.

4. Sciadodendron excelsum. Vista ecuatorial, grano acetolizado.

5. Oreopanax liebmannii. Vista ecuatorial, grano acetolizado.

6. Aralia regeliana. Vista ecuatorial, grano acetolizado. 
BOLETÍN DE LA SOCIEDAD BOTÁNICA DE MÉXICO No. 45, 1983
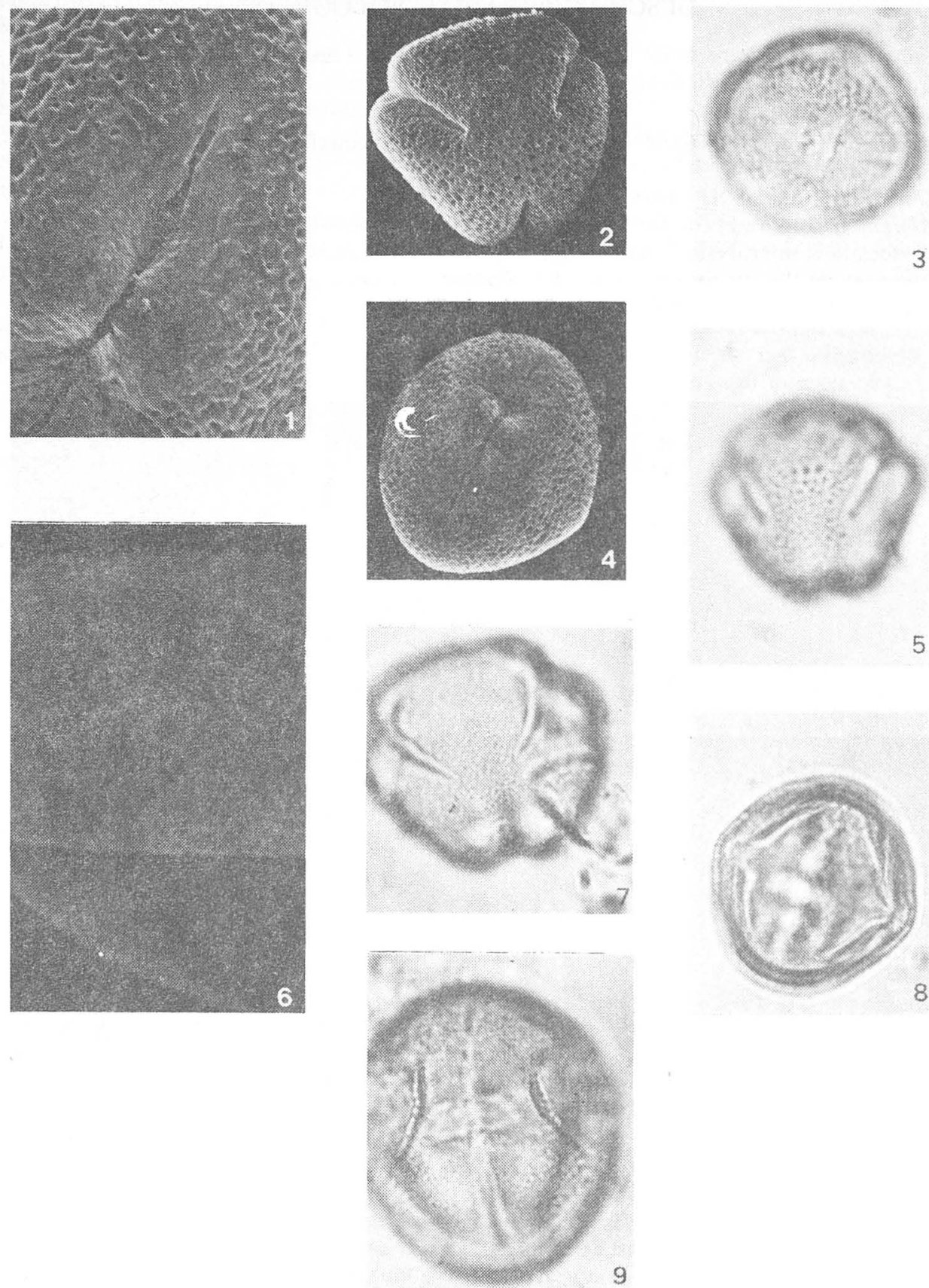

8 


\section{PALINOLOGÍA DE ARALIÁCEAS}

\section{Medidas:}

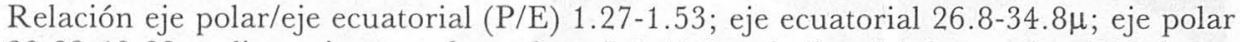

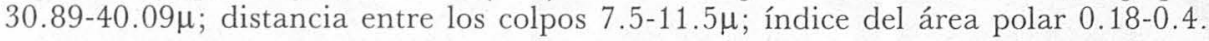

\section{Dendropanax Decne. \& Planchon}

Especies estudiadas;

D. arboreus (L.) Decne. \& Planchon (Figs. 1-5), D. gonatopodus (Donn. Smith) A.C. Smith (Figs. 6-9), D. populifolius (Marchal) A.C. Smith (Figs. 10-14).

Granos eumónadas, de simetría radial, isopolares.

Abertura tricolporada con un colpo transversal bien desarrollado o ausente. Exina tectado perforada, diámetro de las perforaciones de 0.4 a $2 \mu$, los muros simples, su grosor de 2.4 a $6 \mu$. Perímetro en vista polar subangular.

Forma sub-prolada, prolado esferoidal, subesferoidal y oblado esferoidal.

\section{Medidas:}

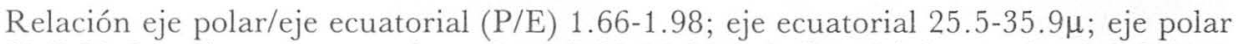

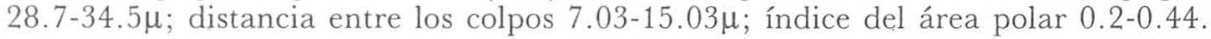

Didymopanax Decne. \& Planchon

Lámina IV

Especie estudiada:

D. morototoni (Aublet) Decne. \& Planchon (Figs. 1-4).

Granos eumónadas de simetría radial, isopolares.

Abertura tricolporada con un colpo transversal pobremente desarrollado. Exina semitectada, las lúminas sin ninguna distribución precisa en el grano, de alrededor de $0.8 \mu$, los muros simples, alrededor de $0.5 \mu$ y, su grosor de $2.4 \mu$. Perímetro en vista polar subangular.

Forma prolado subesferoidal, o subprolada.

\section{Medidas:}

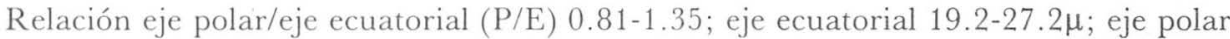

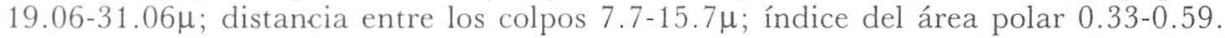

Lámina II. Figuras (Fotografías al MEB y al ML):

1. Aralia regeliana. MEB, vista ecuatorial, con acercamiento a la zona del colpo, grano acetolizado.

2. A. regeliana. MEB, vista polar, grano acetolizado.

3. A. regeliana. ML, vista ecuatorial, grano acetolizado.

4. A. regeliana. MEB, vista ecuatorial, grano acetolizado.

5. A. regeliana. ML, vista polar, grano acetolizado.

6. A humilis. MEB, vista ecuatorial, acercamiento para mostrar la exina, grano sin acetolizar.

7. A. scopulorum. ML, vista polar, grano acetolizado.

8. A. regeliana. ML, vista ecuatorial, en sección óptica, grano acetolizado.

9. A. scopulorum. ML, vista ecuatorial, grano acetolizado. 
BOLETÍN DE LA SOCIEDAD BOTÁNICA DE MÉXICO No. 45, 1983
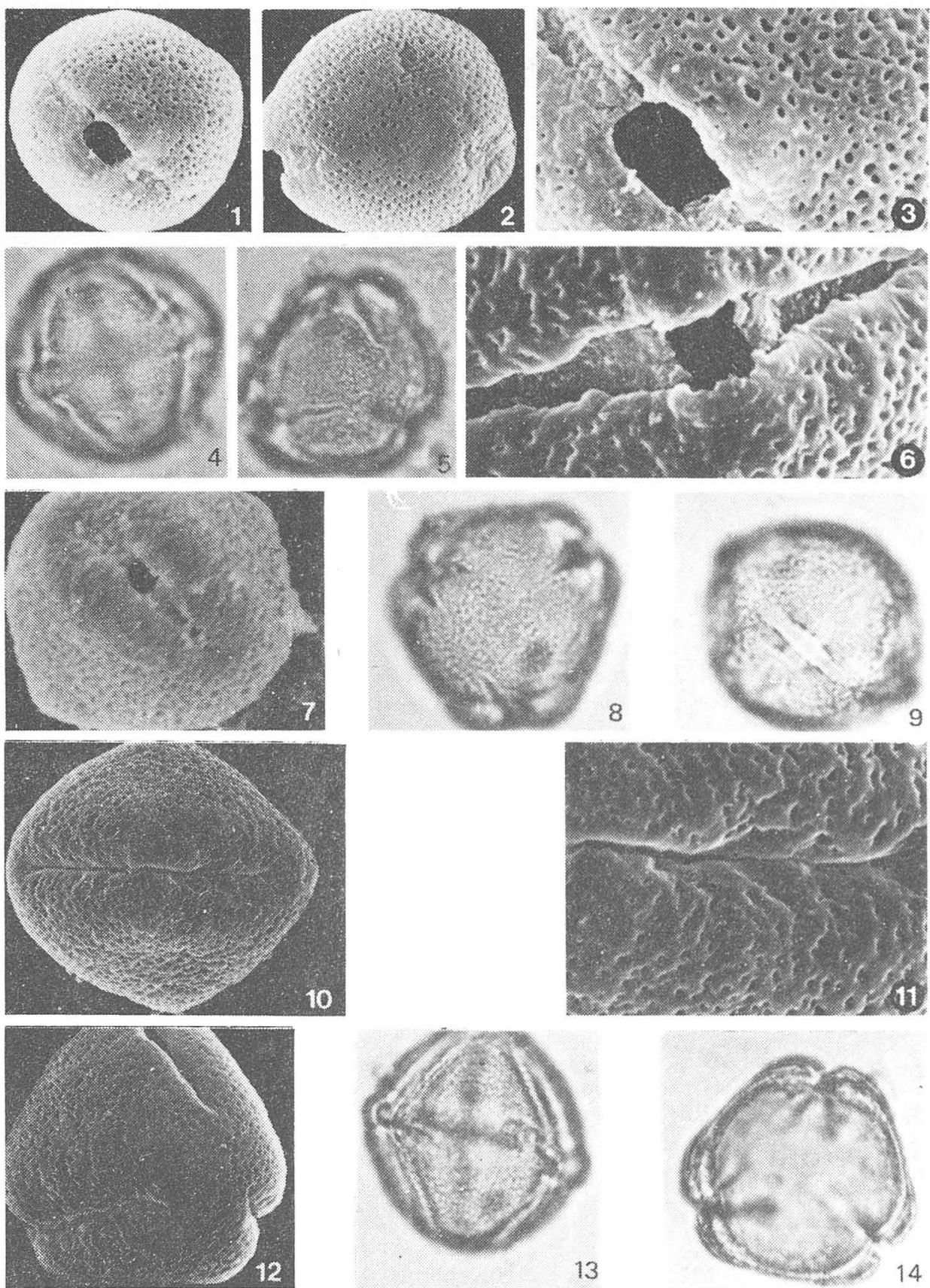


\section{PALINOLOGÍA DE ARALIÁCEAS}

Hedera L.

Especie estudiada:

H. helix L. (Figs. 5-9).

Granos eumónadas, de simetría radial, isopolares.

Abertura tricolporada con un colpo transversal bien desarrollado. Exina semi-tectada, margo-colpado con lúminas en varios tamaños con una forma de polítono irregular, hasta de $3.2 \mu$ de diámetro, los muros simples y duplibaculados, hasta $2 \mu$, su grosor de $2.4 \mu$. Perímetro en vista polar subangular.

Forma prolada o subesferoidal.

Medidas:

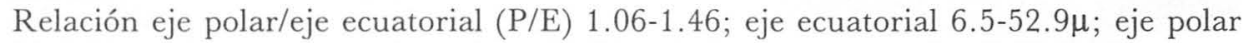

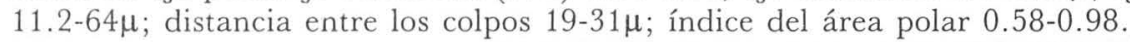

Lámina III. Figuras (Fotografías al MEB y al ML):

1. Dendropanax arboreus. MEB, vista ecuatorial, grano acetolizado.

2. D. arboreus. MEB, vista polar, grano acetolizado.

3. D. arboreus. $\mathrm{MEB}$, vista ecuatorial, acercamiento a la zona dél colpo, grano acetolizado.

4. D. arboreus. ML, vista ecuatorial, grano acetolizado.

5. D. arboreus. ML, vista polar, grano acetolizado.

6. D. gonatopodus. MEB, vista ecuatorial, con acercamiento a la zona del colpo, grano acetolizado.

7. D. gonatopodus. MEB, vista ecuatorial, grano sin acetolizar.

8. D. gonatopodus. ML, vista polar, grano acetolizado.

9. D. gonatopodus. ML, vista ecuatorial, grano acetolizado.

10. D. populifolius. MEB, vista ecuatorial, grano acetolizado.

11. D. populifolius. MEB, vista ecuatorial, con acercamiento a la zona del colpo, grano acetolizado.

12. D. populifolius. MEB, vista polar, grano acetolizado.

13. D. populifolius. ML, vista ecuatorial, grano acetolizado.

14. D. populifolius. ML, vista polar en sección óptica, grano acetolizado.

Lámina IV. Figuras (Fotografías al MEB y al ML):

1. Didymopanax morototoni. $\mathrm{MEB}$, vista ecuatorial, grano acetolizado.

2. D. morototoni. MEB, vista polar, grano acetolizado.

3. D. morototoni. MEB, vista ecuatorial, con acercamiento a la zona del colpo, grano sin acetolizar.

4. D. morototoni. ML, vista ecuatorial en sección óptica, grano acetolizado.

5. Hedera helix. MEB, vista ecuatorial, con acercamiento a la zona del colpo, grano acetolizado.

6. H. helix. MEB, vista polar, grano acetolizado.

7. H. helix. MEB, vista ecuatorial, grano acetolizado.

8. H. helix. ML, vista polar, grano acetolizado.

9. H. helix. ML, vista ecuatorial, grano acetolizado. 
BOLETÍN DE LA SOCIEDAD BOTÁNICA DE MÉXICO No. 45, 1983
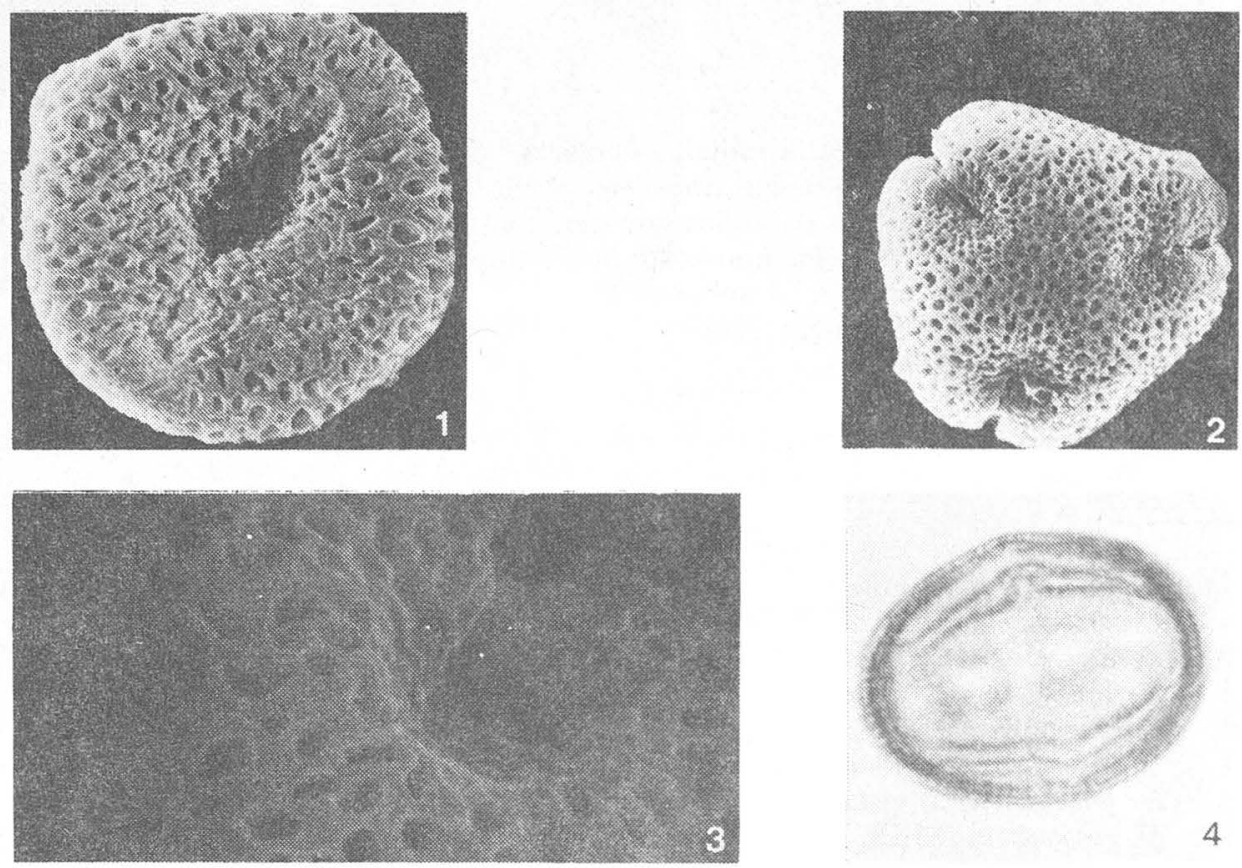

4
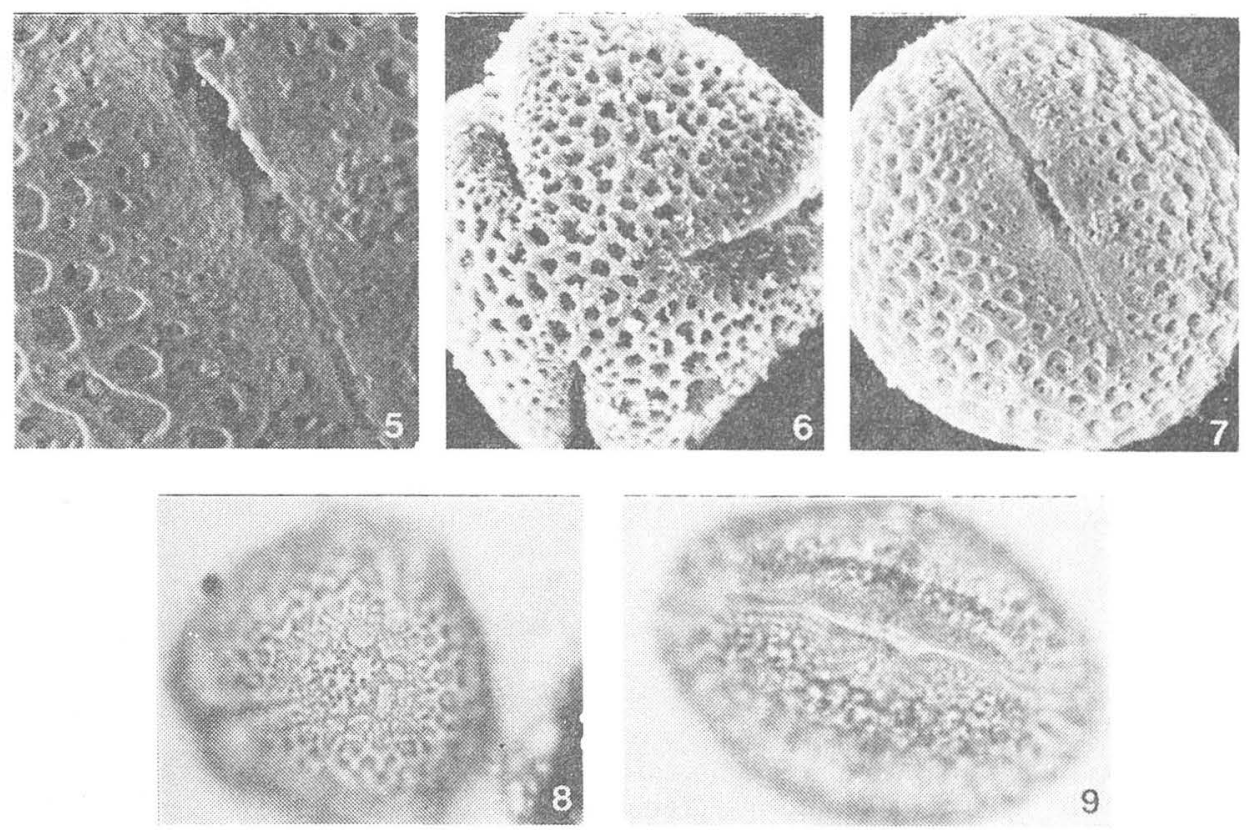


\section{PALINOLOGÍA DE ARALIÁCEAS}
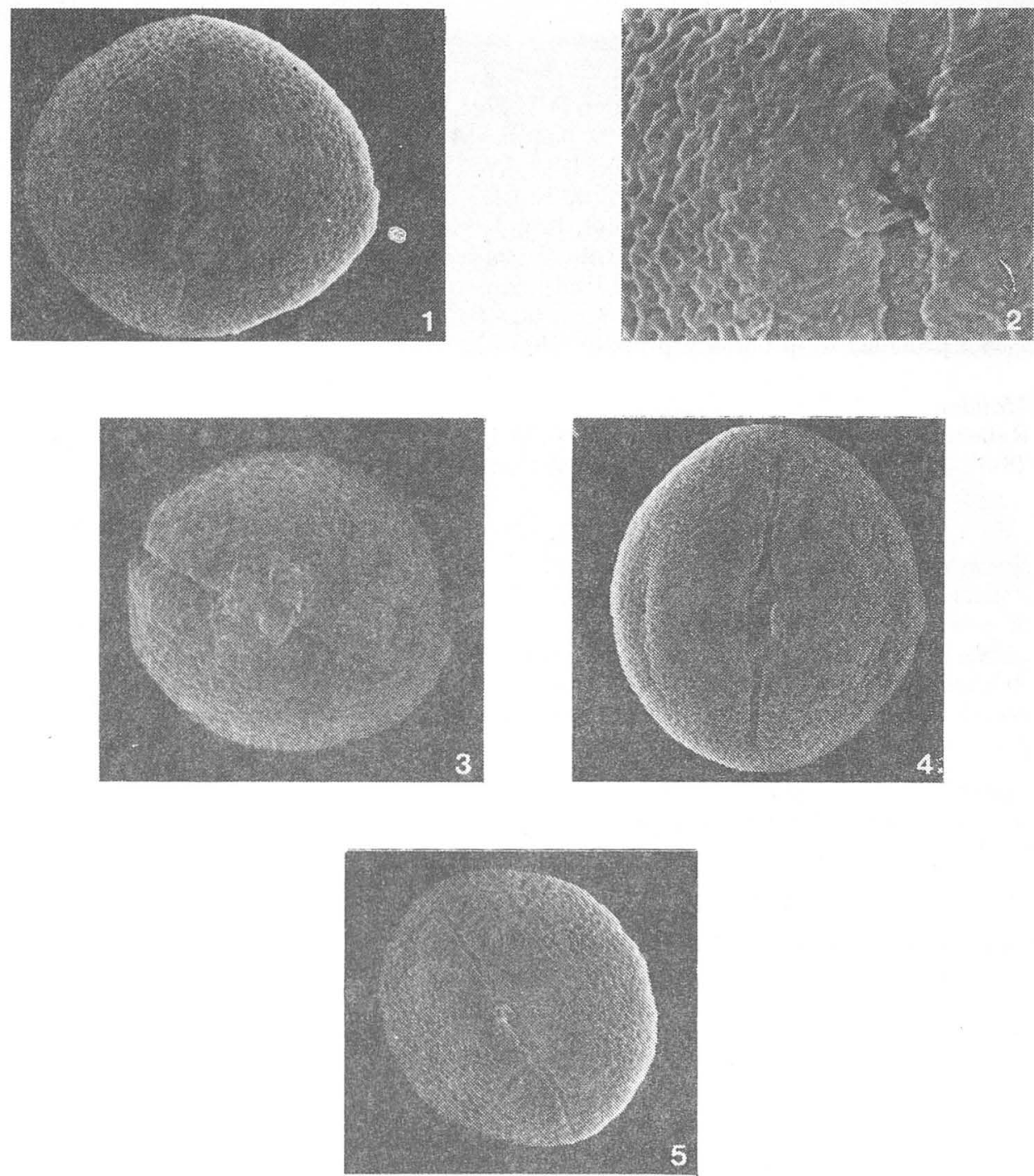

Lámina V. Figuras (Fotografías al MEB):

1. Oreopanax echinops. MEB, vista ecuatorial, grano acetolizado.

2. O. echinops. $\mathrm{MEB}$, vista ecuatorial, con acercamiento a la zona del colpo, grano acetolizado.

3. O. peltatus. MEB, vista ecuatorial, grano sin acetolizar.

4. O. xalapensis. MEB, vista ecuatorial, grano acetolizado.

5. O. flaccidus. MEB, vista ecuatorial, grano acetolizado. 
Oreopanax Decne. \& Planchon

Especies estudiadas:

O. capitatus (Jacq.) Decne. \& Planchon (VI, Figs. 1-5), O. echinops (Schlecht. \& Cham.) Decne. \& Planchon (V, Figs. 1,2), O. flaccidus Marchal (V, Fig. 5), O. liebmannii Marchal (VI, Figs. 11-13), O. obtusifolius L.O. Williams (VI, Figs. 6-10), O. peltatus Linden (V, Fig. 3), O. xalapensis (H.B. \& K.) Decne. \& Planchon (V, Fig. 4).

Granos eumónadas de simetría radial, isopolares.

Abertura tricolporada con un colpo transversal incipiente a más o menos desarrollado. Exina tectado perforada, marco-colpada, diámetro de las perforaciones de 0.8 a $1.6 \mu$, los muros simples, su grosor de $2.4 \mu$ a $6 \mu$. Perímetro en vista polar subangular.

Forma prolada, subprolada y prolado esferoidal.

Medidas:

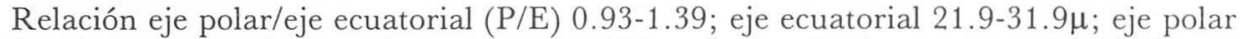

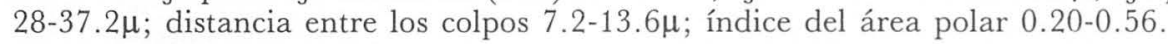

Sciadodendron Griseb.

Lámina VII

Especie estudiada:

S. excelsum Griseb. (Figs. 1-5)

Granos eumónadas, de simetría radial, isopolares.

Abertura tricolporada. Exina tectado perforada, diámetro de las perforaciones de alrededor de $2 \mu$, los muros simples, su grosor de $4 \mu$. Perímetro en vista polar subangular. Forma subprolada o prolado esferoidal.

\section{Medidas:}

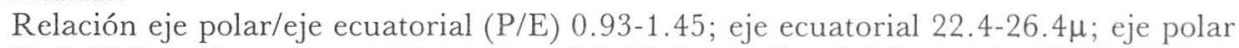
25.2-33.2 $\mu$; distancia entre los colpos 6.5-10.5 ; índice del área polar 0.17-0.41.

Lámina VI. Figuras (Fotografías al MEB y al ML):

1. Oreopanax capitatus. MEB, vista ecuatorial, con acercamiento a la zona del colpo, grano acetolizado.

2. O. capitatus. MEB, vista ecuatorial, grano acetolizado.

3. O. capitatus. $\mathrm{MEB}$, vista polar, grano acetolizado.

4. O. capitatus. ML, vista polar, grano acetolizado.

5. O. capitatus. ML, vista ecuatorial en sección óptica, grano acetolizado.

6. O. obtusifolius. ML, vista ecuatorial, grano acetolizado.

7. O. obtusifolius. ML, vista polar, grano acetolizado.

8. O. obtusifolius. MEB, vista ecuatorial, con acercamiento a la zona del colpo, grano acetolizado.

9. O. obtusifolius. MEB, vista polar, grano acetolizado.

10. O. obtusifolius. MEB, vista ecuatorial, grano acetolizado.

11. O. liebmannii. MEB, vista ecuatorial, con acercamiento a la zona del colpo, grano acetolizado.

12. O. liebmannii. MEB, vista ecuatorial, grano acetolizado.

13. O. liebmanni. MEB, vista polar, grano acetolizado. 
PALINOLOGÍA DE ARALIÁCEAS
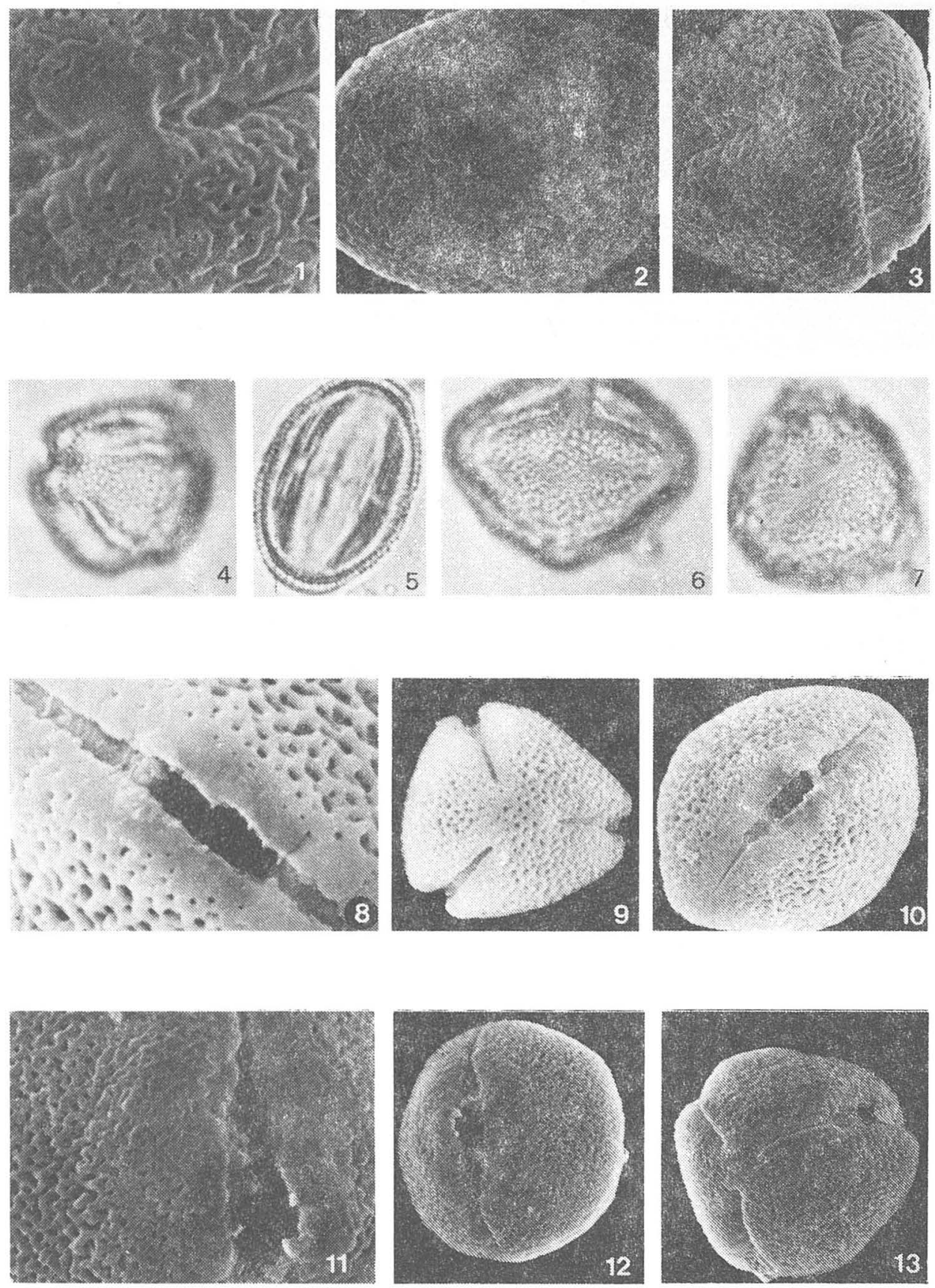

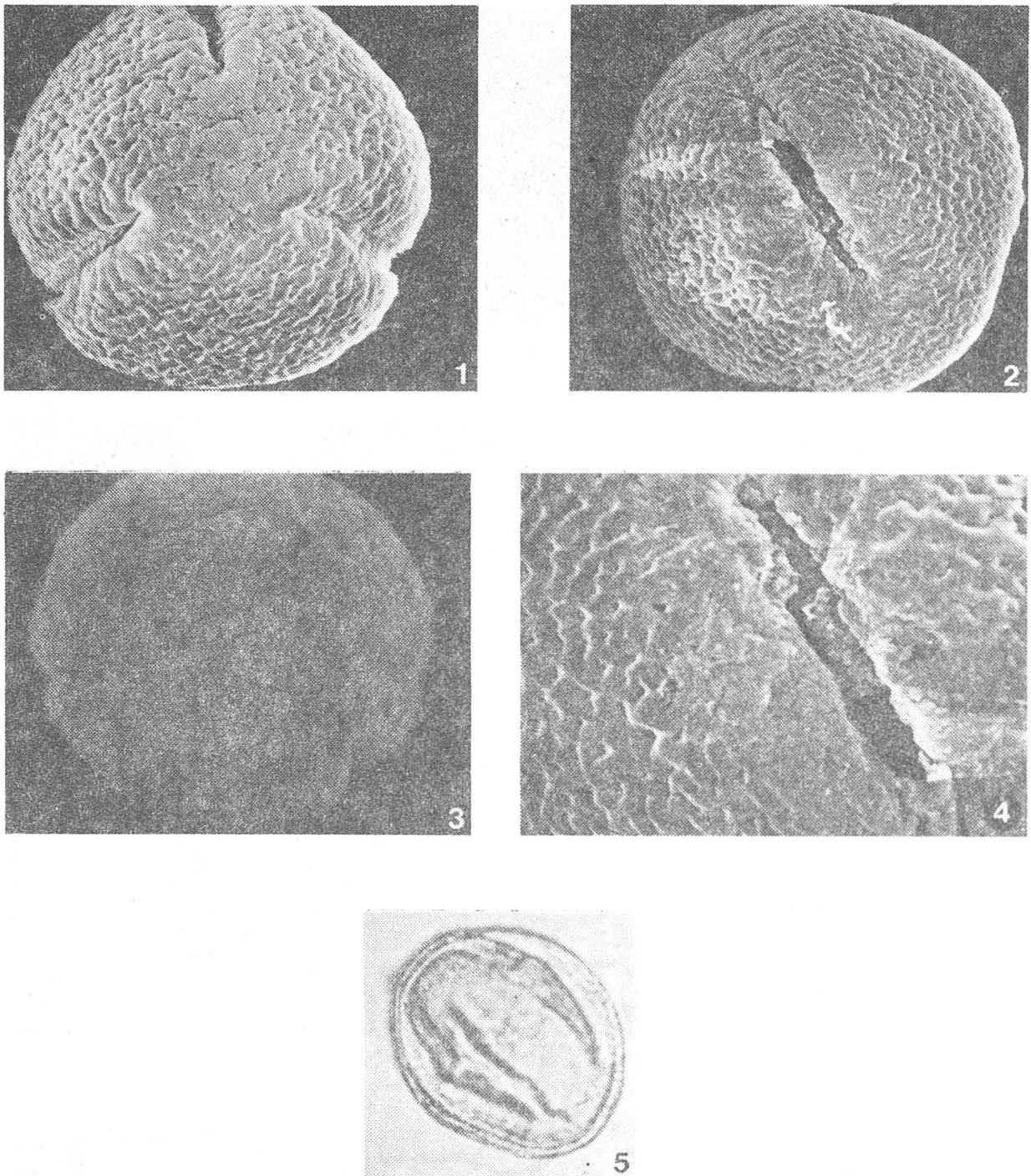

Lámina VI. Figuras (Fotografías al MEB y al ML):

1. Sciadodendron excelsum. MEB, vista polar, grano acetolizado.

2. S. excelsum. MEB, vista ecuatorial, grano acetolizado.

3. S. excelsum. MEB, vista polar, grano sin acetolizar.

4. S. excelsum. MEB, vista ecuatorial, con acercamiento a la zona del colpo, grano acetolizado.

5. S. excelsum. ML, vista ecuatorial en sección óptica, grano acetolizado. 


\section{DISCUSIÓN}

En términos generales la morfología de los granos de polen de las Araliaceae varía poco a nivel genérico, y al específico existen menos diferencias aún, por lo que es difícil reconocerlos tan sólo por sus características palinológicas.

En Hedera helix (hiedra) se advierte una gran variación en el tamaño de los granos. Se observaron dos tamaños; unos muy pequeños, con un eje polar de $25.6 \mu$ y el ecuatorial de $22.4 \mu$, mientras que los mayores alcanzaban medidas del eje polar de $52 \mu$ y el ecuatorial $40.8 \mu$, respectivamente.

Las diferencias principales entre los granos de polen se encontraron especialmente en caracteres de la exina. Los de Dendropanax y Didymopanax no son granos margocolpados. Sciadodendron es apenas margo-colpado y Oreopanax, Aralia y Hedera si son definitivamente margo-colpados.

En cuanto a características de la exina Hedera y Didymopanax tienen granos de polen claramente semi-tectados, mientras que el resto de los géneros son tectado-perforados.

Hedera es el único de los géneros que tiene muros de la exina, que pueden ser duplibaculados; sus perforaciones tienen la forma de polígonos irregulares, características que no comparte con el resto de los granos de polen de otras araliáceas.

Desde el punto de vista de la morfología floral, Eyde y Tseng (1971) estudiaron cerca de 150 especies de araliáceas, concluyendo que las más primitivas son aquellas que tienen flores polímeras, inflorescencias paniculadas y hojas pinnadas. Tseng (1973) afirma también que un tipo de polen generalizado entre las araliáceas primitivas es aquel pequeño y esferoidal y que dichos caracteres son compartidos por varios taxa, con hojas palmadamente lobadas o compuestas.

Si seguimos las conclusiones a las que llegaron Eyde y Tseng, Sciadodendron y Aralia que tienen flores polímeras, hojas pinnadas e inflorescencias paniculadas presentan también características palinológicas primitivas, como son una exina tectado-perforada, (la cual según Walker y Doyle (1975), es una etapa anterior a la exina semi-tectada) y una forma del grano de polen prolado-esferoidal.

Oreopanax, que también presenta estas características palinológicas tiene, sin embargo, algunas otras morfológicas más avanzadas porque sus especies pueden tener hojas simples o compuestas, inflorescencias paniculadas o racemoso-capitadas y sus flores casi siempre son pentámeras. Dendropanax se encuentra en un estado intermedio por que las hojas son simples; sus flores pentámeras o polímeras y las inflorescencias umbelas simples o paniculadas.

Hedera y Didymopanax, tienen características palinológicas más avanzadas, por presentar granos de polen con exina semi-tectada, forma desde subprolado hasta proladoesferoidal; hojas simples o palmado-compuestas, flores pentámeras e inflorescencias umbeladas.

Estos caracteres palinológicos en unión de otros, deberán tomarse en cuenta para afinar y cambiar la clasificación de las Araliaceae, ya que, como afirma Graham (1966), el grupo se dividió basándose en caracteres poco satisfactorios como son el tipo de prefloración y forma de los pétalos; y en otras clasificaciones, por características anatómicas.

\section{AGRADECIMIENTOS}

A la M. en C. Beatríz Ludlow-Wiechers, la revisión del manuscrito y sus sugerencias para la realización de este trabajo. Al doctor Enrique Martínez-Hernández su guía 
en el inicio del trabajo. A los biólogos Luis Roldán Ramos, José Luis Alvarado y Mónica Ayala, del Laboratorio de Palinología del INIREB su desinteresada ayuda. Al técnico Tiburcio Láez su ayuda con las muestras de microscopio electrónico de barrido. Al doctor Alan Graham su colaboración en la obtención de bibliografía. Al doctor Luis Cendrero y a la señora Beatríz Gómez Varela la lectura del manuscrito.

\section{BIBLIOGRAFÍA}

CHAO, C.Y. 1954. Comparative pollen morphology of the Cornaceae and allies. Taiwania 5:93-106.

CHAZARO, M. 1982. Flora apícola de la zona cafetalera de Coatepec, Ver. pp. 95-103. En: E. Jiménez-Ávila, y A. Gómez-Pompa (Eds.) Estudios ecológicos en el agroecosistema cafetalero. INIREB-CECSA, México.

ERDTMAN, G. 1943. An introduction to pollen analysis. Ronald Press Co. New York.

1972. Pollen morphology and Plant Taxonomy. Angiosperms 1. Hafner Publishing Company. New York.

EYDE, R.H. y C.C. TSENG. 1971. ¿What is the primitive floral structure of Araliaceae? J. Arnold Arbor. 52:205-239.

GRAHAM, S.A. 1966. The genera of Araliaceae in the Southeastern United States. J. Arnold Arbor 47:126-136.

GRAHAM, A. y D.M. JARZEN. 1969. Studies in Neotropical Paleobotany. 1. The Oligocene communities of Puerto Rico. Ann. Missouri Bot. Gard. 56:308-357.

GRUAS-CAVAGNETTO, C. y N. BUI. 1976. Présence de Pollen d'araliacées dans le palógene anglais et francais. Review of Paleobotany and Palynology 22:61-72.

KREMP, G.O.W. 1965. Morphological encyclopedia of Palynology. The University of Arizona Press. Tucson. 263 pp.

MULLER, J. 1981. Fossil pollen records of extant angiosperms. Bot. Rev. 47(1):10-145.

PALACIOS, R. 1968. Morfología de los granos de polen de árboles del estado de Morelos. An. Esc. Nac. Cienc. Biol. Méx. 16:41-169.

RADULESCU, D. 1974. Contribution a l'etude de la morphologie du pollen des familles Cornaceae et Araliaceae. Acta Botanica Horti Bucurestiensis 1974:125-131.

SMITH, A.C. 1944. Araliaceae. North American Flora. New York Botanical Garden, New York. 28B:3-41.

SOSA, V. 1979. Araliaceae. En: A. Gómez-Pompa (Ed.) Flora de Veracruz. Instituto Nacional de Investigaciones sobre Recuros Bióticos. Xalapa, Ver. 7:38 pp.

STRAKA, H. y A. SIMON. 1967. Araliaceae. pp. 430-431. En: Palynologia Madagassica et Mascarenica. Pollen et Spores 9(3):427-466.

TSENG, CC. 1973. Systematic palynology of Tupidanthus and Plerandra (Araliaceae). Grana 13:51-56.

WALKER, J.W. \& J.A. DOYLE. 1975. The bases of Angiosperm phylogeny: Palynology. Ann. Missouri Bot. Gard. 62:664-723. 